\title{
The Effect of Skill-based Sexual Enhancement Counseling Program in Quality of life in Women with Multiple Sclerosis-a quasi-experimental study
}

\author{
Negin Sayari \\ Arak University of Medical Sciences \\ Katayon Vakilian ( $\nabla$ dr.kvakilian@arakmu.ac.ir) \\ Arak University of Medical Sciences \\ Zohre Khalajinia \\ Qom University of Medical Sciences and Health Services \\ Seyyed Amir Hejazi \\ Qom University of Medical Sciences and Health Services \\ Mostafa Vahedian \\ Qom University of Medical Sciences and Health Services
}

\section{Research article}

Keywords: Sexual health, Multiple sclerosis, Reproductive health, Quality of life

Posted Date: August 19th, 2020

DOI: https://doi.org/10.21203/rs.3.rs-42866/v1

License: (c) (1) This work is licensed under a Creative Commons Attribution 4.0 International License. Read Full License 


\section{Abstract}

Background: Multiple sclerosis (MS) is one of the world's most common neurologic disorders and the leading cause of neurologic disability in young adults. This study aimed to investigate skill-based sexual enhancement counseling on Quality of Life (QLI) of women with Multiple Sclerosis.

Methods: the present study is a quasi-experimental design with two groups. Forty-two patients with multiple sclerosis and their spouses $(\mathrm{N}=88)$ voluntarily participated in this study based on the inclusion and exclusion criteria. After obtaining the written consent forms, the couples were randomly assigned in two groups. The subjects in the intervention group ( $\mathrm{N}=22$ couples) received the skill-based sexual enhancement counseling program in six sessions (each for 90 minutes). The control group just received routine medical procedure during this period. Both groups completed three stages of pretest-posttest, and the standard MSQOL54 questionnaire 3 months later. Data were analyzed by SPSS-20 using descriptive and inferential statistics (repeated measures ANOVA, chi-square, Friedman and t-test).

Results: The results showed that the participants' overall quality of life in the intervention group in pretest was $55.32 \pm 9.140$ which increased to $73.89 \pm 13.39$ in posttest and to $92.59 \pm 20.45,3$ months later. Also, the physical quality of life showed a significant difference between the two groups 3 months after the intervention $(P<0.05)$. There was a significant difference between the two groups in psychological quality of life, too. The subscales of physical and mental QLI such as sexual satisfaction, sexual function, emotional wellbeing, social function and energy in post-test and follow-up were significantly different $(P<0.05)$.

Conclusions: According to the findings, skill-based sexual enhancement counseling program is effective in quality of life enhancement. Therefore, it is recommended to health care providers to use enhancement program alongside the other rehabilitation and medical services to improve the patients' quality of life.

\section{Background}

Multiple sclerosis (MS) is one of the world's most common neurologic disorders and the leading cause of neurologic disability in young adults (1). MS is a chronic, progressive disease that causes injury to the myelin sheath of the central nervous system. It is a long-lasting disease that can affect the brain, spinal cord, and the optic nerves. These physical complications affect patients' social life, family, quality of life, work, and health $(2,3)$. A survey in 104 countries showed that the number of people suffering with the disease had been increased from 2.1 million in 2008 to 2.3 million in 2013(1). In Iran, one study reported the incidence of this disease at the range of 6.02 in 2013 and 3.87 per 100,000 people in 2014(4). Today, the life expectancy of patients with multiple sclerosis is 8 to 10 years less than the normal population and women are more likely to live longer than men (5). The mean age at disease onset is 28.54 years old in Iran (6). This age range is often considered as the best period of fertility and intimate relationship for women. Most women at this age range get marry and accept social and family responsibilities (7). Physicalpsychological and medicine-related complications etc. fatigue, muscle weakness, changes in menstruation, pain, anxiety, depression, urinary incontinence, and intestinal problems decrease chance of independence and ability to effectively participate in the society and interfere with ability to work, pursue leisure activities, and carry on usual life roles of employment $(8,9)$. Physical, psychological and social effects have a significant impact on the quality of life and health of these patients $(10,11)$. Dealing with the unique physical and psychological stressors associated with being in close contact (living with or caring for) a loved one with MS, marital quality is considered to be an important factor(12) .Lower relationship satisfaction, relationship distress, sexual dysfunction, and low quality of life are often reported by the couples, one of whom is affected by MS, as compared to normal couples(12,13).

According to the results of a study, using communication skills, conflict resolution skills, and relationship in a 8-hour programming via in-person workshops disseminated across the teleconferences meeting improved the quality of life of these patients. Although rare studies conducted on effectiveness of relationship enrichment program on quality of life in MS patients, this research was conducted to assess the effect of Skill-based Sexual Enhancement Counseling Program(SSE-CP) on the quality of life.

\section{Methods}

\section{Study design}


This research was a quasi-experimental study with pretest-posttest and follow-up design with control group.

\section{Setting}

The target population consisted of women with MS referring to MS Society of Qom province between 2018 and 2019. Qom is the seventh largest metropolis of Iran and is located $140 \mathrm{~km}$ to the south of Tehran with estimated 1,201,158 populations.

This research was conducted in MS Society of Qom. Multiple Sclerosis Society of Iran (MS Association of Iran) is a non-political, non-commercial, non-profit and charitable organization. The Association provides medical, educational, and welfare services to these patients with a population over 1310 patients.

\subsection{Sample size}

The sample size was calculated using Cochran formula considering $95 \%$ and $80 \%$ power coefficients, and the variance (14), accordingly 22 couples were placed in each group.

\section{Patients}

The patients were invited to participate in the study through written posters on social networks including telegram and Instagram pages of MS society of Qom city. 44 cases (out of 65 volunteers) who met the inclusion criteria signed the written consent forms after getting aware of the study's goals and ethical considerations. Inclusion criteria included age over 18 years old, being married with at least two years of marital life, diagnosed as MS patient according to McDonald's criteria by a neurologist, having a history of treatment for at least six months, absence of any severe marital discord and court cases, no drug addiction, absence of any recent life crises other than multiple sclerosis, and absence of any psychiatric disorder. The exclusion criteria were Absence of one spouse for more than one session, unwillingness to continue the project, recurrence of the illness, and the concurrent presence in another study. In this study, 65 subjects volunteered to participate in the study, of whom 44 cases met the criteria.

The MS patients and their spouses (44 couples were randomly divided into two groups. The assigned block "A" or "B" was determined based on the letter "A" or "B" hidden in 44 sealed and unmarked envelopes. The assigned group was identified when each participant opened her picked envelope. Finally, 20 couples in the intervention group and 21 couples in the control group were reminded for the analysis (Fig. 1).

\section{Study tools}

The participants were assessed by the questionnaire of demographic characteristics (including age, sex, education, spouse age, spouse illness, number of children, duration of illness, and any other illness), and 54-item MS Quality of Life Questionnaire (MSQOL-54). MSQOL-54 was designed by Vickrey (1995) via adding 18 items to the short-form quality of life questionnaire (SF36). Minimum and maximum score is between 0-100, in that higher score indicates higher quality of life (QLI).

The general dimensions included physical health and psychological health. The subscales of physical health were: physical function (10 items scoring with a 3-option Likert scale), role limitations due to physical problems ( 4 items scoring as yes $=1$, no $=$ 2), health perceptions (5 items scoring with a 5-option Likert scale), energy/fatigue (5 items scoring with a 6-option Likert scale), pain (3 items scoring with a 6-option Likert scale), sexual function (4 items scoring with a 4-option Likert scale), and social function (3 items scoring with a 5-option Likert scale).

psychological dimension subscales included: health distress (4 items scoring with a 6-option Likert scale), emotional well-being (5 items scoring with a 6-option Likert scale), role limitations- emotional ( 3 items scoring as yes $=1$, no $=2$ ), cognitive function ( 4 items scoring with a 6-option Likert scale).

Finally, 4 items in overall health change (1 item in 0-100), overall sexual satisfaction ( 1 item from 0-100), and overall quality of life ( 2 items in 1-7) and structural validity were confirmed using factor analysis. The Cronbach's alpha coefficient of 0.82 was reported for this questionnaire (15).

\section{Methods of study}

Page $3 / 12$ 
Intervention Skill-based Sexual Enhancement Counseling Program (SSE-CP) of couples was performed in six 90-minute sessions in MS Association. The SSEP protocol was administered for couple counseling in the presence of the couples who were blind of the presence of other couples in the research group. SSE-CP meeting protocol was designed based on similar interventions extracted from textbooks and articles $(16,17)$.

The designed protocol was reviewed and confirmed by experts including a clinical psychologist, a sexologist, family counselors, and neurologists. The experts' recommendations regarding sexual difficulties and needs of MS patients were included in the protocol. The couple therapy sessions of SSE-CP were held in MS Association of Qom city twice a week, each for 90 minutes. One of researchers as a counselor was trained a 3-month course of SSE-CP. In the first session, the participants were explained about goals, expectations and procedure of the study. Couples who were willing to participate in the project and met the criteria signed the consent forms and completed the questionnaires including demographic characteristics, (MSQOL-54). The sessions were administered face to face with each couple under supervision of a fertility health PhD professional for six 90-minute sessions twice a week. The participants were assessed by (MSQOL-54) at the end of the sixth session. They were asked to use the skills they were trained in daily life and contact with researcher after 12 weeks in the same place to complete (MSQOL-54). The content of sessions was as following:

The contents of the skill-based sexual enhancement counseling sessions were as follows:

Session 1: building rapport, reviewing the complications caused by MS on marital and sexual relationships, and presenting the sexual response pattern and its changes in Multiple Sclerosis. The assigned homework was reading booklet of sexual functions and sexual positions in MS patients.

Session 2: problem- and conflict-solving methods and skills to make personal changes (for both the participants). Checking the homework assigned in the previous session.

Session 3: training empathy skills, self-emotional expression skill, displaying a video clip for enhancement emotional-sexual expression; homework: talk about a sexual or marital problem and explain if they could solve it, how they solved it, and how they dealt with it (by using the learned skills

Session 4: reviewing the content and the homework of the previous session. Training negotiation skill.

Session 5: training effective spouse communication skills by displaying video clip.

Session 6: providing counseling for specific sexual problems in Multiple Sclerosis patients.

The obtained data were analyzed using SPSS-20 software. Mean and standard deviation were used to describe the descriptive analysis, and the independent t-test, ANOVA repeated measurement, T-test, Friedman and Chi-square tests were used for analytical analysis.

\section{Results}

Demographic findings indicated that the mean age of participants in the intervention group was $37.75 \pm 4.19$, and $39.95 \pm 3.48$ in the control group, showing no significant difference. There was no significant difference between the mean ages of spouses in the intervention group (42.25 \pm 55.21 ) and the control group (43.17 \pm 3.68$)$. The duration of disease ranged between 1 to 11 years with the mean $2.94 \pm 4.55$ in the intervention group) and $5.67 \pm 2.67$ in the control group, indicating no significant difference $(P<0.05)$.

Findings showed that there was a significant difference between the quality of life of patients in the intervention and control groups $(p<0.05)$. The results show that the physical dimension of the quality of life increased from pretest to post test and 12 weeks after the intervention in the intervention group as compared to the control group. In contrast, in control group, the mean of physical dimension QLI in posttest and 3 months later showed no change. Moreover, the psychological QLI dramatically increased in posttest and 3 months after counseling. Based on Eta coefficients, the intervention explained $46 \%$ of the variance of changes in the quality of life (Table 1). Generally, the overall QLI significantly between two groups and QLI more than in intervention group in after intervention and 3 months later than control group. Other results are presented in (Table 1). 
Table 1

Demographic characters of patients in intervention and control groups

\begin{tabular}{|c|c|c|c|c|}
\hline \multirow[t]{2}{*}{ variable } & & \multirow{2}{*}{$\begin{array}{l}\text { Intervention } \\
\mathrm{N}(\%)\end{array}$} & \multirow{2}{*}{$\begin{array}{l}\text { Control } \\
\mathrm{N}(\%)\end{array}$} & \multirow[t]{2}{*}{ P Value } \\
\hline & & & & \\
\hline \multirow[t]{3}{*}{ education } & diploma & $4(20)$ & $7(33.3)$ & \multirow[t]{3}{*}{$0.59 *$} \\
\hline & Bachelor & $12(57.1)$ & $13(65)$ & \\
\hline & Master/PhD & $2(9.5)$ & $3(15)$ & \\
\hline \multirow[t]{4}{*}{ Number of children } & 0 & $4(20)$ & $1(4.8)$ & \multirow[t]{4}{*}{$0.47 *$} \\
\hline & 1 & $7(35)$ & $10(47.6)$ & \\
\hline & 2 & $8(40)$ & $9(42.9)$ & \\
\hline & 3 & $1(5)$ & $1(4.8)$ & \\
\hline \multirow{3}{*}{$\begin{array}{l}\text { Disease duration } \\
\text { (Year) }\end{array}$} & $1-5$ & $13(65)$ & $13(61.9)$ & \multirow[t]{3}{*}{$0.85^{\star}$} \\
\hline & $6-10$ & $6(28.6)$ & $6(30)$ & \\
\hline & Above11 & $1(5)$ & $1(4.8)$ & \\
\hline Disease duration & Mean \pm SD & $4.55 \pm 2.94$ & $5.33 \pm 2.67$ & $0.37 * \star$ \\
\hline age & Mean \pm SD & $37.75 \pm 3.48$ & $39.95 \pm 3.48$ & $0.07 * \star$ \\
\hline Age of spouse & Mean \pm SD & $42.25 \pm 5.21$ & $43.17 \pm 3.68$ & $0.30 * \star$ \\
\hline Marriage duration & Mean \pm SD & $8.45 \pm 2.94$ & $7.33 \pm 3.67$ & 0.16 ** \\
\hline
\end{tabular}

Table 2

Comparison Psychological, Physical and Overall QLI in intervention and control groups

\begin{tabular}{|c|c|c|c|c|c|c|c|c|c|c|}
\hline \multicolumn{2}{|l|}{ Quality of life } & Mean & $\begin{array}{l}\text { Std. } \\
\text { Deviation }\end{array}$ & Mean & $\begin{array}{l}\text { Std. } \\
\text { Deviation }\end{array}$ & Mean & $\begin{array}{l}\text { Std. } \\
\text { Deviation }\end{array}$ & $\begin{array}{l}\text { *P } \\
\text { value } \\
\text { group }\end{array}$ & $\begin{array}{l}\text { Eta } \\
\text { coefficient }\end{array}$ & $F$ \\
\hline & & \multicolumn{2}{|c|}{ Pre intervention } & \multicolumn{2}{|c|}{ Post intervention } & \multicolumn{2}{|c|}{3 months later } & & & \\
\hline \multirow[t]{2}{*}{ Psychological } & Intervention & 33.65 & 7.56 & 42.89 & 9.85 & 53.32 & 11.62 & 0.001 & 0.378 & 23.67 \\
\hline & control & 31.90 & 7.66 & 34.44 & 8.54 & 34.43 & 7.85 & & & \\
\hline \multirow[t]{2}{*}{ Physical } & Intervention & 21.67 & 5.20 & 30.99 & 8.31 & 39.27 & 9.90 & 0.008 & 0.422 & 28.43 \\
\hline & control & 19.35 & 6.72 & 21.18 & 6.80 & 22.59 & 9.81 & & & \\
\hline \multirow[t]{2}{*}{ Overall QLI } & Intervention & 55.32 & 9.140 & 73.89 & 13.39 & 92.59 & 20.45 & 0.001 & 0.466 & 34.05 \\
\hline & control & 51.25 & 10.387 & 55.63 & 11.27 & 57.03 & 16.22 & & & \\
\hline
\end{tabular}

The results of the subscale of quality of life in physical dimension showed that the physical and social performance of the intervention group increased significantly and there was a significant decrease in the fatigue subscale in this group, as compared to the control group. Other variables are presented in Table 3. The results of the subscales of psychological dimension indicated that emotional well-being increased significantly immediately and 3 months after the intervention in the experimental group (Table 3). 
Table 3

Comparison subgroups QLI in intervention and control groups

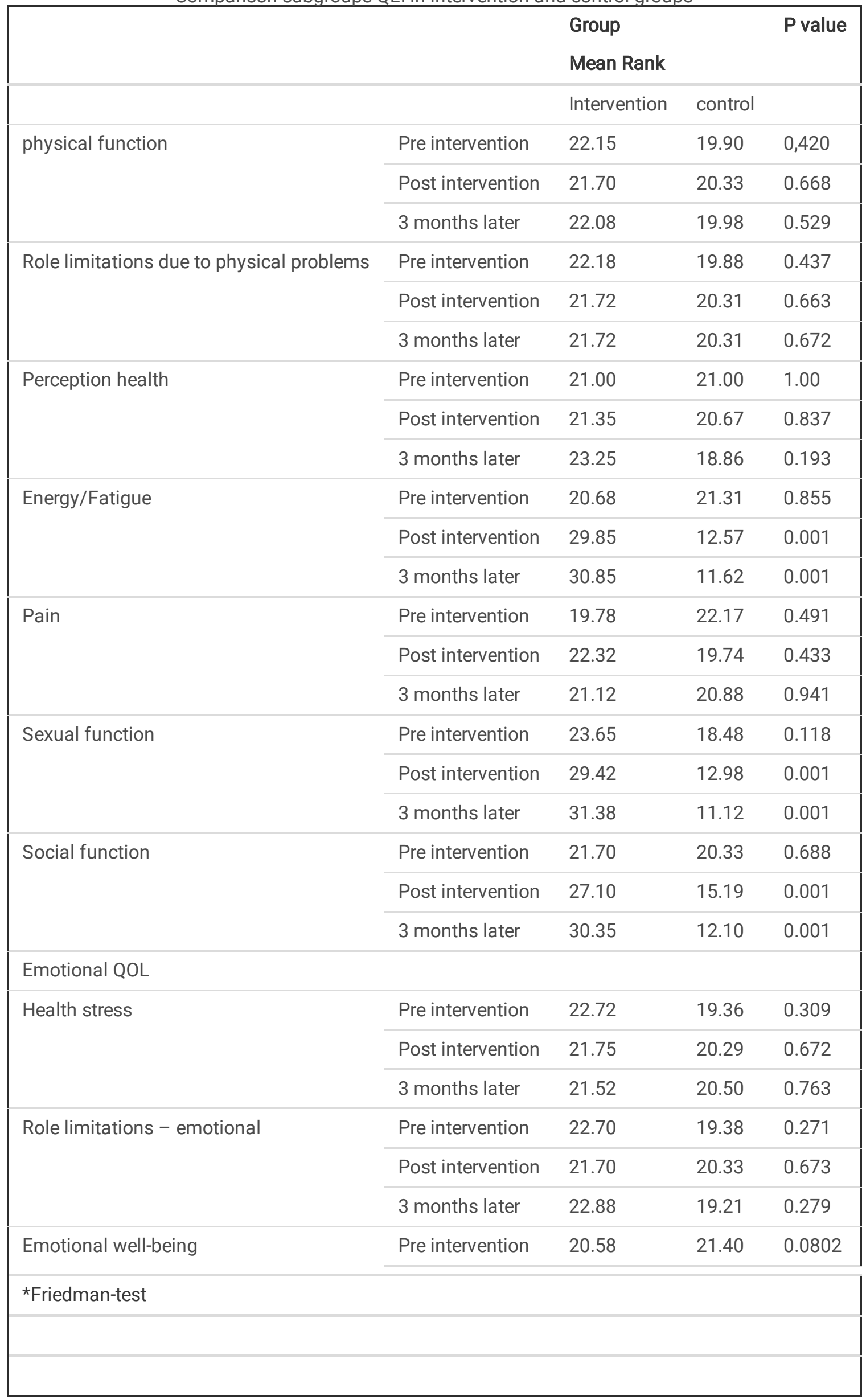




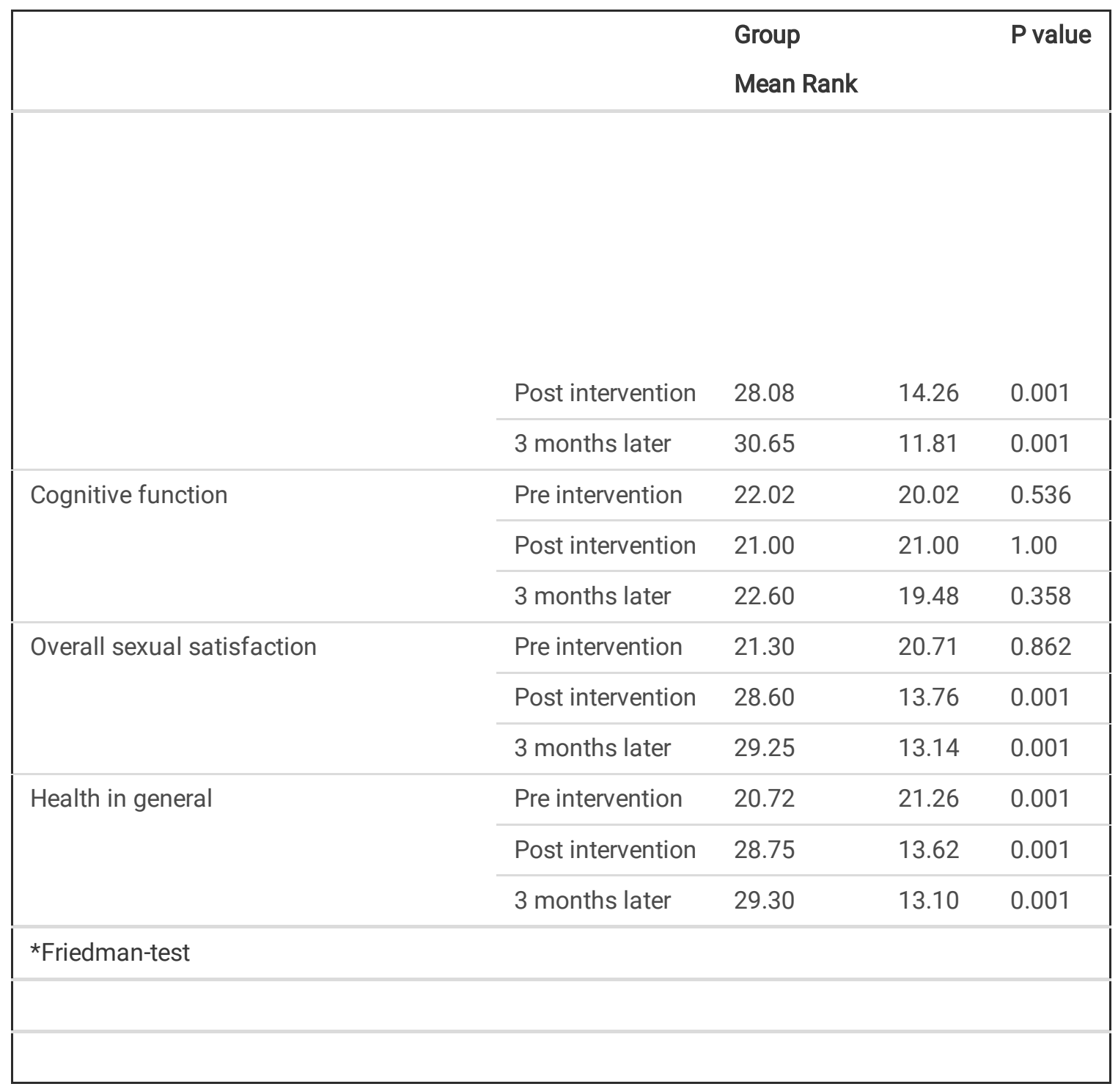

\section{Discussion}

Many researchers have identified inadequacy in effective communication and constructive conflict-solving as main barriers to couple communication, which consequently leads to reduced relationship satisfaction $(18,19)$. Eliminating such barriers can improve the couple communication and their social interactions and increase the quality of life (20). The results of this study showed that quality of life increased through training conflict-solving skills, empathy, effective communication, emotion express, problem-solving, and negotiation skill. The couples, during the intervention, could gain information and awareness about the impact of MS on marital issues and women's sexual phases. As a result, their quality of life improved. This intervention not only improved the participants' physical dimension, as it reduced the feeling of fatigue and improved the patients' social performance and sexual function, but also improved their psychological dimension, as it improved their feeling of emotional well-being. The results showed that the effect of this intervention was stable and continued 3 months after the intervention as well. Other studies also revealed the effectiveness of skill-based sexual counseling (18). Nathan etal. conducted a research aiming to investigating the effectiveness of marital enrichment on normal couples. They trained couple communication enrichment to 34 couples for 11 hours. The results showed that couples' sexual function increased and this change was stable to three months after the intervention though there could be found no significant change in marital satisfaction (21).

Another quasi-experimental study was conducted in 2016 on 80 spouses of MS patients in Iran. The results showed that five sessions of couple relationship enrichment program increased the quality of marital relationship for spouses of patients with MS (18). 
Sexual function is a complex process that affects physical, psychological, and intrapersonal factors. A study in Iran showed that $82 \%$ of women with MS have sexual dysfunction. Therefore, it is of great importance to provide training and counseling programs to resolve their functional disorder (22). The questionnaire of MS patients' quality of life, in this study, showed that sexual satisfaction is one of the sexual quality components; in this context, the emphasis of training skills, in this study, was on improving the sexual health of these couples. Conflict-solving skills, self-expression, effective communication, and sexual training all were used to improve sexual satisfaction. The results of the present study indicated that the women's sexual satisfaction increased immediately after the intervention, and even it continued 3 months later. Further, studies have been conducted on the effect of skillbased sexual counseling on the quality of life, and the results revealed the effectiveness of such intervention on different aspects of quality of life. Tomkins et al. investigated the effectiveness of relationship enrichment program on the quality of life in MS patients. The participants in this study were 3002 subjects, of which 2498 subjects received relationship enrichment program in one-day workshop for 8 hours. 504 subjects received one to two hours of telephone counseling for 8 sessions and the other subjects were in the control group. 3 months later, the results showed that the quality of life in relation to the patients' health improved after participating in the counseling sessions. Conflict-solving skill and the couples' relationship improved(12) .

The results of the study by Ysrraelit et al. indicated that more than half of MS patients reported low quality of life, especially in physical dimensions (23). The results found by Alhazzani, et al. showed that MS patients have low quality of life and thus, programs are needed to improve their conditions(24). To explain this finding, it can be said that multiple sclerosis is known as a debilitating disease with side effects, medications, physical and psychological changes, social problems, anxiety due to disease management, recurrence anxiety, occupational problems, and factors like these cause persistent and chronic stress that can reduce the patient's personal energy to deal with problems, increase perceived pain and interfere with physical and psychological roles and consequently, reduce quality of life. Making changes in these variables can contribute to improving quality of life.

One quasi-experimental study was carried out to examine the effect of sex therapy on the QOL of 30 women with MS. The participants in the treatment group received 12 weekly sessions of sex therapy, while those who were in the control group received no treatment. The results showed improvements in the different aspects of sexual function such as desire, arousal, lubrication, orgasm, satisfaction, and pain, satisfaction with sexual function, overall quality of life, energy, improved cognitive function, improved sexual function, and improved social function as compared to the control group (25).

The results of the study by Parva et al. showed that the enrichment program had positive effects on sexual boldness and improved the participants' sexual function. Empathy and emotional self-expression skills, negotiation skills, problem-solving, intimate couple training, and effective spouse communication skills are skills to improve sexual intimacy, and thereby contributing to sexual satisfaction (26).

Due to psychological and emotional factors, women with MS feel that they lost their sexual attraction and are unable to have sex, make love (touch, hug, etc.) and ultimately have no sexual intimacy with their husbands. This issue is especially true in cultures where attractiveness is associated with beauty, health and good appearance. Meanwhile, having an intimate relationship and the ability to express emotions can reduce these irrational beliefs in this group of women and help them to increase their desire for sexual intimacy. The participants were trained, during the sessions of marital relationship enrichment, skills that help satisfy basic family needs such as love, kindness, belonging, trust, loyalty, security, and pleasure. Compassionate sensitivity can be the most important factor in improving security and validating couple's relationship and creating constructive intimacy and healthy atmosphere for personal growth (18).

Quality of life as a multidimensional variable is directly correlated with a variety of positive variables such as marital satisfaction (27), sexual satisfaction (28), and family relationships (29).

The other results of the present study showed a significant increase in the patients' emotional well-being, which was stable to three months after the intervention.

Emotional self-expression skill motivated the patients to express their emotions and discuss about it. Talking about emotions helps the patient to empty his/her destructive emotions and does not destroy the individual's self-esteem. It also increases the power of endurance in the patient and helps the patient to behave more adaptive in dealing with marital problems and keep his/her sexual self-esteem $(30,31)$. 
It seems that although sex therapy had an impact on the quality of life and psychological aspects of the MS patients, it did not significantly influence their health perception, pain and physical limitations because of the physical disabilities and dysfunctions that they experience.

It seems that rehabilitation programs, such as aerobic exercise and physical therapy make an improvement in the quality of life, especially in physical dimension $(32,33)$.

This study involved some limitations such as small sample size and different levels of the disease. On the other hand, this study had strengths such as face to face training, presence of husband with his wife and assessing counseling 3 month later.

\section{Conclusion}

The rehabilitation programs that are designed for patients with MS should contain the relevant skill-based sexual enhancement programs to improve the quality of the patients' marital relationship (including their sexual relationship and satisfaction). In this context, it can contribute to improving the quality of life of patients and their spouses. Furthermore, the results of the present study can help the patients with MS or other chronic diseases to improve the quality of their marital relationship.

\section{Abbreviations}

Multiple sclerosis(MS)

Quality of Life (QLI)

Skill-based Sexual Enhancement Counseling Program (SSE-CP)

54-item MS Quality of Life Questionnaire (MSQOL-54)

\section{Declarations}

\section{Ethics approval and consent to participate}

This research is based on a MSc dissertation with the ethical code IR.ARAKMU.REC.1397.350 in Arak University of Medical Sciences. The research project was approved by Amir Kabir Clinical Research Center. All participants were verbally consented prior to participating in this study and then signed inform consent.

\section{Consent for publication}

Not applicable

\section{Availability of data and materials}

The data that support the findings of this study are available from Arak University of Medical Sciences but restrictions apply to the availability of these data, which were used under license for the current study, and so are not publicly available. Data are however available from the authors upon reasonable request and with permission of Arak University of Medical Sciences.

\section{Competing interests}

The authors have no competing interests to declare.

\section{Funding}

The proposal of this study was funded by Arak university of Medical Sciences by grant no:3155

\section{Authors' contributions}


NS and KV designed the study. SA H and NS helped to doing sampling. Z KH and MV and KV wrote the first draft of the manuscript. $\mathrm{KV}$ and SA H contributed to subsequent drafts and have reviewed and agreed with the content of the final manuscript. MV analyzed the data. $\mathrm{Z} \mathrm{KH}$ and $\mathrm{KV}$ wrote the final draft. It is necessary to mention that all authors have read and approved the manuscript.

\section{Acknowledgments}

The authors would like to thank the esteemed staff of the Multiple Sclerosis Society of Qom city for their sincere cooperation. We also thank the patients who participated in the study.

\section{Authors' Information}

Negin Sayari, , MSC candidate in Midwifery Counseling, Arak University of Medical Sciences, School of medicine, Arak, Iran.

* Katayon Vakilian(Corresponding Author): Associated professor in Reproductive Health, Department of midwifery, Arak University of Medical Sciences, Arak, Iran

Zohre Khalajinia, , Assistant professor in Reproductive Health, Department of Nursing Midwifery, School of nursing and midwifery, Qom University of Medical Sciences. Qom, Iran.

Seyyed Amir Hejazi, Assistant professor in Neurolog, Neuroscience Research Center, Qom University of Medical Sciences, Qom, Iran, Mostafa Vahedian, Assistant professor in Epidemiology, Neuroscience Research Center, Qom University of Medical Sciences, Qom, Iran,

\section{References}

1. Browne P, Chandraratna D, Angood C, Tremlett H, Baker C, Taylor BV, et al. Atlas of multiple sclerosis 2013: a growing global problem with widespread inequity. eurology.14;83(11):1022-4. DOI: https://doi.org/10.1212/WNL.0000000000000768

2. Jennum P, Wanscher B, Frederiksen J, Kjellberg J. The socioeconomic consequences of multiple sclerosis: a controlled national study. European Neuropsychopharmacology. 2012;22(1):36-43. https://doi.org/10.1016/j.euroneuro.2011.05.001

3. Kołtuniuk A, Przestrzelska M, Karnas A, Rosińczuk J. The Association Between Sexual Disorders and the Quality of Life of Woman Patients With Multiple Sclerosis: Findings of a Prospective, Observational, and Cross-Sectional Survey. Sexual Medicine. 2020;8(2: 297-306 https://doi.org/10.1016/j.esxm.2020.02.009

4. Eskandarieh S, Heydarpour P, Elhami S-R, Sahraian MA. Prevalence and incidence of multiple sclerosis in Tehran, Iran. Iranian journal of public health. 2017;46(5):699.

5. Lunde HMB, Assmus J, Myhr K-M, Bø L, Grytten N. Survival and cause of death in multiple sclerosis: a 60-year longitudinal population study. J Neurol Neurosurg Psychiatry. 2017;88(8):621-5. http://dx.doi.org/10.1136/jnnp-2016-315238

6. Heydarpour P, Khoshkish S, Abtahi S, Moradi-Lakeh M, Sahraian MA. Multiple sclerosis epidemiology in Middle East and North Africa: a systematic review and meta-analysis. Neuroepidemiology. 2015;44(4):232-44.

7. Landfeldt E, Castelo-Branco A, Svedbom A, Löfroth E, Kavaliunas A, Hillert J. The long-term impact of multiple sclerosis on the risk of divorce. Multiple sclerosis and related disorders. 2018;24:145-50. https://doi.org/10.1016/j.msard.2018.07.002

8. Strober LB, Christodoulou C, Benedict RH, Westervelt HJ, Melville P, Scherl WF, et al. Unemployment in multiple sclerosis: the contribution of personality and disease. Multiple Sclerosis Journal. 2012;18(5):647-53.

https://doi.org/10.1177/1352458511426735

9. Wendebourg MJ, Heesen C, Finlayson M, Meyer B, Pöttgen J, Köpke S. Patient education for people with multiple sclerosisassociated fatigue: a systematic review. PLoS One. 2017;12(3). https://doi.org/10.1371/journal.pone.0173025

10. Campbell J, Rashid W, Cercignani M, Langdon D. Cognitive impairment among patients with multiple sclerosis: associations with employment and quality of life. Postgraduate Medical Journal. 2017;93(1097):143-7.

http://dx.doi.org/10.1136/postgradmedj-2016-134071

11. Brola W, Sobolewski P, Fudala M, Flaga S, Jantarski K. Multiple sclerosis: patient-reported quality of life in the Świętokrzyskie Region. Medical Studies/Studia Medyczne. 2017;33(3):191-8. DOI: https://doi.org/10.5114/ms.2017.70345

Page $10 / 12$ 
12. Tompkins SA, Roeder JA, Thomas JJ, Koch KK. Effectiveness of a relationship enrichment program for couples living with multiple sclerosis. International journal of MS care. 2013;15(1):27-34.

13. Kessler TM, Fowler CJ, Panicker JN. Sexual dysfunction in multiple sclerosis. Expert review of neurotherapeutics. 2009;9(3):341-50.

14. Sanders AS, Foley FW, LaRocca NG, Zemon V. The multiple sclerosis intimacy and sexuality questionnaire-19 (MSISQ-19). Sexuality and Disability. 2000;18(1):3-26. https://doi.org/10.1023/A:1005421627154

15. Qaderi K, Merghati KE. Female sexual problem in multiple schlorosisi and its association with quality of life Journal of Nursing and Midwifery Urmia University of Medical Sciences 2013;11(7):1-11.

16. Masoumi SZ, Kazemi F, Nejati B, Parsa P, Karami M. Effect of Sexual Counseling on Marital Satisfaction of Pregnant Women Referringto Health Centers in Malayer (Iran): An educational randomized experimental study. Electronic physician. 2017;9(1):3598.

17. Aghausofi A, Tarkhan M, karimi r. Effect of Enrichment Education of Communication on Conjugal Satisfaction of Addicted Couples. Clinical Psychology \& Personality. 2018;16(1):49-56.

18. Navidian A, Navabi Rigi S, Imani M, Soltani P. The effect of sex education on the marital relationship quality of pregnant women. Hayat. 2016;22(2):115-27[persian]

19. Asadi ZS, Sadeghi R, Taghdisi MH, Zamani-Alavijeh F, Shojaeizadeh D, Khoshdel AR. Sources, Outcomes, and Resolution of Conflicts in Marriage among Iranian women: A qualitative study. Electronic physician. 2016;8(3):2057.

20. Ahmadi K, Rezazade M, Saadat H, Kimiaei SA, Zade NH. Contribution of marital conflict to marital quality in short and longterm marriages: An actor-partner interdependence model. Journal of education and health promotion. 2015;4.

21. Nathan EP, Joanning HH. Enhancing marital sexuality: An evaluation of a program for the sexual enrichment of normal couples. Journal of sex \& marital therapy. 2012;11(3):157-64.https://doi.org/10.1080/00926238508405441

22. Dehghan-Nayeri N, Khakbazan Z, Ghafoori F, Nabavi SM. Sexual dysfunction levels in iranian women suffering from multiple sclerosis. Multiple sclerosis and related disorders. 2017;12:49-53. https://doi.org/10.1016/j.msard.2017.01.005

23. Ysrraelit MC, Fiol MP, Gaitán MI, Correale J. Quality of life assessment in multiple sclerosis: different perception between patients and neurologists. Frontiers in neurology. 2018;8:729. https://doi.org/10.3389/fneur.2017.00729

24. Alhazzani AA, Alqahtani MS, Alahmari MS, Asiri MA, Alamri NM, Sarhan LA, et al. Quality of life assessment among multiple sclerosis patients in Saudi Arabia. Neurosciences (Riyadh, Saudi Arabia). 2018;23(2):140-7.

25. Zamani M, Tavoli A, Khasti BY, Sedighimornani N, Zafar M. Sexual therapy for women with multiple sclerosis and its impact on quality of life. Iranian journal of psychiatry. 2017;12(1):58.

26. Parva M, Lotfi R, Nazari MA, Kabir K. The effectiveness of sexual enrichment counseling on sexual assertiveness in married women: A randomized controlled trial. Shiraz E-Medical Journal. 2018;19(1):e14552. doi: 10.5812/semj.14552.

27. Gharibi M, Sanagouymoharer G, Yaghoubinia F. The relationship between quality of life with marital satisfaction in nurses in social security hospital in Zahedan. Global journal of health science. 2016;8(2):178. doi: 10.5539/gjhs.v8n2p178

28. Gillespie BJ. Correlates of sex frequency and sexual satisfaction among partnered older adults. Journal of sex \& marital therapy. 2017;43(5):403-23.https://doi.org/10.1080/0092623X.2016.1176608

29. Aguiar K, Marson F, Gomez C, Pereira M, Paschoal I, Ribeiro A, et al. Physical performance, quality of life and sexual satisfaction evaluation in adults with cystic fibrosis: An unexplored correlation. Revista Portuguesa de Pneumologia (English Edition). 2017;23(4):179-92.https://doi.org/10.1016/j.rppnen.2017.02.009

30. Ansari N, Vakilian K, Mehrabi Rezveh F. Sexual health during menopause-counselling with an approach to emotional regulation on sexual satisfaction. Family Medicine \& Primary Care Review. 2020;21(4):318-

23.https://doi.org/10.5114/fmpcr.2019.90159

31. Mikolajczak M, Luminet O. Trait emotional intelligence and the cognitive appraisal of stressful events: An exploratory study. Personality and individual differences. 2008;44(7):1445-53.https://doi.org/10.1016/j.paid.2007.12.012

32. Niwald M, Redlicka J, Miller E. The effects of aerobic training on the functional status, quality of life, the level of fatigue and disability in patients withmultiple sclerosis-a preliminary report. Aktualności Neurologiczne. 2017;17(1). 
33. Rakhshan M, Ganjalivand S, Zarshenas L, Majdinasab N. The Effect of Collaborative Care Model-Based Intervention on Hope in Caregivers and Patients with Multiple Sclerosis: A Randomized Controlled Clinical Trial. International journal of community based nursing and midwifery. 2018 Jul;6(3):218.

\section{Figures}
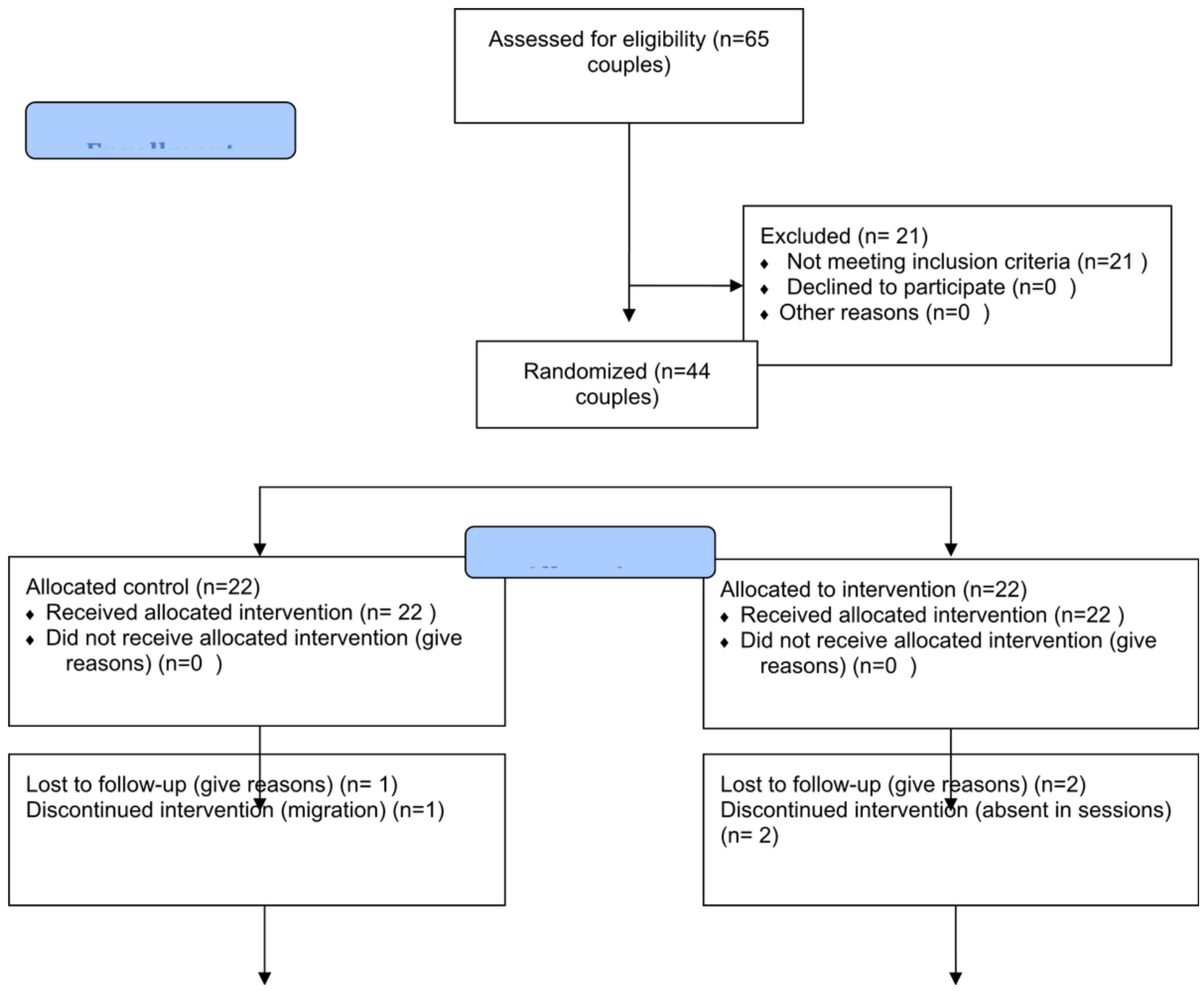

Figure 1

Study chart 